\title{
A Study on Self-Esteem, Locus of Control and Achievement Motivation among High School Students
}

\author{
Jing Lv ${ }^{1}$, Ben-fa Wang ${ }^{2}$ \\ 1) School of education and psychology, Unversity of Jinan, Shandong, China \\ ${ }^{2)}$ School of education and psychology, Unversity of Jinan, Shandong, China
}

\begin{abstract}
To understand current high school students' self-esteem, locus of control and achievement motivation and research the relationship between them, we investigated on 270 high school students by using SES, IPC and AMS. The results are as follows: (1)There is no significantly gender difference in self-esteem, locus of control and achievement motivation.. (2) Self-esteem, locus of control and achievement motivation among high school students were significantly correlated. (3) Self-esteem can predict achievement motivation of high school students in locus of control for mediation.
\end{abstract}

Keywords-Achievement motivation., Self-esteem, Locus of control, High school students

\section{高中生自尊、心理控制源与成就动机的关系研究 \\ 吕晶 王本法}

济南大学教育与心理科学学院, 济南, 山东, 中国

摘 要 为了解高中生自尊、心理控制源与成就动机的一般现状, 初步探讨高中生自尊、心理控制源与成就动机之间的关系, 采用 自尊量表 (SES)、内在性、有势力的他人及机遇量表（IPC）和成就动机量表（AMS）对270名济南市某重点高中高一学生进行调查。 结果表明:（1）高中生自尊、心理控制源和成就动机在性别上均无显著差异。(2) 高中生自尊、心理控制源与成就动机之间存在显著 的相关。（3）高中生的自尊能够预测其成就动机，并以心理控制源作为中介。

关键词 成就动机，自尊，心理控制源，高中生

自尊是一个人在对待自己的态度中表现出的对自我价 值的判断，是个体对自我价值的判断和对自己持有的肯定 或否定的情感体验。国内外学者对自尊的具体研究主要集 中在自尊与学业成绩的关系、自尊与人际关系等方面。研 究发现自尊与学业成绩密切相关。

Rotter 提出心理控制源是指人们日常生活中对行为或 事件结果的一般性看法。早期的关于心理控制源的研究表 明, 心理控制源与其他变量之间存在关系，外控倾向与焦 虑、抑郁情绪有关。

成就动机是人们追求高目标, 完成困难任务, 竞争并超 过他人的人格力量。几十年来, 这一领域的研究成果虽然丰 硕庞杂,但其理论倾向主要集中于以下几个方面：（1）麦克 里兰的广泛的社会成就动机理论;（2）阿特金森的“期待
一价值”成就动机理论; (3) 韦纳的成就动机归因理论; (4) 霍纳的女性“避免成功”概念及成就动机;（5）余安邦、杨 国枢的“个我一社会取向成就动机理论”及成就动机的跨文 化研究机制的性差研究。

关于自尊、心理控制源与成就动机三者之间的关系研 究, 李睿等人的研究发现, 大学生成就动机、心理控制源 和自尊三者之间存在不同程度的统计学上的相关。

\section{1. 问题提出}

近年来, 国内外学者从理论和实证等不同角度对成就 动机进行了研究和探索, 并取得了一定的进展。但总的来 说，在我国有关高中生成就动机和心理控制源、自尊的研 究还比较少。我国高中生的成就动机在性别上是否存在差

山东省教育科学规划“教育招生考试科学研究专设课题”: 素质教育与应试教育博交中的高考改革研究（项目标号: S1159）成果之一 
异? 自尊和心理控制源是否会影响成就动机? 为了更深入 的解释出这三者之间的关系, 本研究一方面拟揭示高中生 成就动机、自尊、心理控制源的现状，另一方面拟深入探 讨高中生成就动机、自尊、心理控制源的关系。

\section{2 研究方法}

\section{1 研究对象}

本研究选取济南市某重点中学高一年级 5 个班 300 名 学生进行测试, 回收问卷 271 份, 其中有效问卷 263 份, 男生 125 人 $(47.5 \%)$ ，女生 138 人 $(52.5 \%)$ 。

\section{2 研究工具}

\subsection{1 自尊量表( The Self-Esteem Scale 简称 SES)}

SES 由 Rosenberg 1965 年设计, 用以评定青少年关于 自我价值和自我接纳的总体感受。该量表共包含 10 个项 目, 采用 4 级评分方式, 研究表明, 该量表信、效度良好。

\subsection{2 内在性、他人掌控及机遇量表（Internality，The}

Powerful Others and Chance Scale 简称 IPC)

IPC 量表由 Levenson1981 年编制, 分为内在性, 他人 掌控, 机遇三个分量表。每个分量表有 8 个题目, 共 24 题, 6 级评分, 采用 7 点记分法, 该量表分半信度为 $0.64(\mathrm{P}<$ 0.01 )。Levenson 在 10 多项研究结果的基础上提出了该量 表的常模。

\subsection{3 成就动机量表表 (Achievement Motivation Scale 简称}

AMS )

由挪威心理学家 Gjesme T 和 Nygard R于 1970 年编制。 中文版本为我国研究者叶仁敏和挪威 Hegtvet K A 于 1988 年合作译制该量表分为两部分共 30 个题目, 包括追求成功

（Ms）与避免失败（Mf）2个分量表。

\section{3 研究程序}

向济南市某重点中学高一年级 5 个班发放问卷 300 份, 回收有效问卷 263 份, 问卷的有效回收率为 $87.7 \%$ 。调查 时使用统一的指导语, 由主试进行集体施, 课堂填写完毕后 当场回收问卷。审查, 严格剔除无效问卷。在量表施测的 同时获得被试的性别、年龄等一般人口统计学资料。

\section{3 结果分析}

3.1 自尊、心理控制源与成就动机的性别差异

本研究首先比较了男、女高中生在自尊、心理控制源 各维度、成就动机以及成就动机各维度上的差异, 发现没 有达到统计学上的显著差异。具体平均数和标准差见表 1 。
表1 不同性别高中生自尊、心理控制源与成就动机的独立样本 $\mathrm{t}$ 检验 $(\mathrm{M} \pm \mathrm{SD})$

\begin{tabular}{cccc}
\hline & 男生 $(125)$ & 女生 $(138)$ & $\mathrm{t}$ \\
\hline 自尊 & $30.62 \pm 5.08$ & $30.61 \pm 4.41$ & 0.026 \\
内在性 & $31.88 \pm 6.82$ & $31.80 \pm 6.21$ & 0.925 \\
他人掌控 & $15.06 \pm 7.87$ & $13.62 \pm 7.40$ & 0.129 \\
机遇 & $17.14 \pm 7.50$ & $17.72 \pm 7.17$ & 0.521 \\
追求成功 & $42.45 \pm 8.34$ & $41.37 \pm 6.81$ & 1.153 \\
避免失败 & $35.17 \pm 9.75$ & $36.52 \pm 9.20$ & -1.165 \\
成就动机 & $7.28 \pm 16.27$ & $4.84 \pm 13.28$ & 1.337 \\
\hline
\end{tabular}

注: *: p <0.05; **: p <0.01

3.2 高中生自尊、心理控制源与成就动机的相关分析

表 2 显示, 自尊与内在性和成就动机、追求成功与内 在性和内在性、避免失败与他人掌控和机遇有显著的正相 关关系, 自尊与他人掌控、自尊与机遇、自尊与避免失败、 内在性与避免失败、他人掌控与追求成功、他人掌控与成 就动机、机遇与成就动机呈现显著的负相关, 机遇与追求 成功相关不显著。

表2 自尊、心理控制源与成就动机的相关 $(r)$

\begin{tabular}{|c|c|c|c|c|c|c|c|}
\hline & 自尊 & 内在性 & $\begin{array}{c}\text { 他人掌 } \\
\text { 控 }\end{array}$ & 机遇 & $\begin{array}{c}\text { 追求成 } \\
\text { 功 }\end{array}$ & $\begin{array}{c}\text { 避免失 } \\
\text { 败 }\end{array}$ & $\begin{array}{l}\text { 成就 } \\
\text { 动机 }\end{array}$ \\
\hline 自尊 & 1 & & & & & & \\
\hline 内在性 & $0.436^{* *}$ & 1 & & & & & \\
\hline 他人掌控 & $-0.374 * *$ & $-0.330^{* * *}$ & 1 & & & & \\
\hline 机遇 & $-0.330^{* *}$ & $-0.328^{* *}$ & $0.569^{* *}$ & 1 & & & \\
\hline 追求成功 & 0.321 ** & $0.331^{* *}$ & $-0.178^{* *}$ & -0.117 & 1 & & \\
\hline 避免失败 & $-0.349 * *$ & $-0.328^{* *}$ & $0.360^{* *}$ & $0.339^{* *}$ & $-0.501 * *$ & 1 & \\
\hline 成就动机 & $0.388 * *$ & $0.379^{* *}$ & $-0.321 * *$ & $-0.227 * *$ & $0.833 * *$ & $\frac{-0.896 * *}{}$ & 1 \\
\hline \multicolumn{8}{|c|}{ 注: *: $\mathrm{p}<0.05 ; * *: \mathrm{p}<0.01$} \\
\hline \multicolumn{8}{|c|}{3.3 高中生自尊对成就动机的回归分析 } \\
\hline \multicolumn{8}{|c|}{$\begin{array}{l}\text { 本研究发现, 自尊与成就动机存在一定的相关关系, } \\
\text { 为进一步探讨二者之间的关系, 对自尊和成就动机进行了 } \\
\text { 回归分析。以自尊为自变量, 以追求成功、避免失败和成 } \\
\text { 就动机分别作为因变量, 进行了三次一元线性回归分析, } \\
\text { 对三个回归方程进行方差分析, 三个方程的检验结果均显 } \\
\text { 示回归方程显著, 模型与数据的拟合程度很好。结果说明, } \\
\text { 高中生自尊对成就动机有一点的预测作用, 详见表 } 3 \text { 。 }\end{array}$} \\
\hline
\end{tabular}


表3 高中生自尊对成就动机的回归分析表

\begin{tabular}{ccccc}
\hline & $\mathrm{r}$ & $\mathrm{R}$ 方 & $\mathrm{F}$ & $\mathrm{t}$ \\
\hline 追求成功 & 0.321 & 0.103 & $29.964 * *$ & $5.474 * *$ \\
避免失败 & 0.349 & 0.122 & $36.232 * *$ & $-6.019 * *$ \\
成就动机 & 0.388 & 0.150 & $46.176 * *$ & $6.795 * *$ \\
\hline
\end{tabular}

注: *: p <0.05; **: p <0.01

3.4 高中生自尊对心理控制源的回归分析

本研究发现, 自尊与心理控制源的三个维度存在一定 的相关关系，为进一步探讨二者之间的关系，对自尊和心 理控制源进行了回归分析。以自尊为自变量, 以内控性、 他人掌控和机遇分别作为因变量, 进行了三次一元线性回 归分析, 对三个回归方程进行方差分析, 三个方程的检验 结果均显示回归方程显著, 模型与数据的拟合程度很好。 结果说明, 高中生自尊对心理控制源有一定的预测作用, 详见表 4 。

表4 高中生自尊对心理控制源的回归分析表

\begin{tabular}{ccccc}
\hline & $\mathrm{r}$ & $\mathrm{R}$ 方 & $\mathrm{F}$ & $\mathrm{t}$ \\
\hline 内控性 & 0.436 & 0.190 & $61.419 * *$ & $7.837 * *$ \\
他人掌控 & 0.373 & 0.139 & $42.236^{* *}$ & $-6.499 * *$ \\
机遇 & 0.330 & 0.109 & $31.969^{* *}$ & $-5.654 * *$ \\
\hline
\end{tabular}

注: *: p <0.05; **: p <0.01

\section{5 心理控制源对成就动机的回归分析}

以成就动机为因变量, 以心理控制源的三个维度内控 性, 他人掌控和机遇为分别为自变量, 对成就动机的回归 分析结果如如下图所示, 三组回归方程均差异显著。详见 表 5。

表5 高中生心理控制源对成就动机的回归分析表

\begin{tabular}{lllll}
\hline & $\mathrm{r}$ & $\mathrm{R}$ 方 & $\mathrm{F}$ & $\mathrm{t}$ \\
\hline 内控性 & 0.379 & 0.144 & $43.887 * *$ & $6.625 * *$ \\
他人掌控 & 0.321 & 0.103 & $30.024 * *$ & $-5.479 * *$ \\
机遇 & 0.277 & 0.077 & $21.640 * *$ & $-4.652 * *$ \\
\hline
\end{tabular}

注: *: p <0.05; **: p <0.01

\section{6 以心理控制源为中介的路径分析模型}

由于自尊，心理控制源和成就动机三个变量之间的相 关程度都很高, 且回归效应显著, 考虑到自尊对成就动机 的预测是受心理控制源的中介作用的影响，为探讨心理控
制源是否对自尊和成就动机之间起中介的作用, 所以对三 者进行路径分析。结合本研究获得的相关矩阵和回归分析 结果, 利用 amos 软件进行路径分析, 经过反复验证和分析 后, 得出自尊、心理控制源和成就动机的关系模型, 模型 拟合的基本指数为卡方 $=38.739, \mathrm{p}<0.01, \mathrm{GFI}=0.947$, $\mathrm{NFI}=0.898, \mathrm{CFI}=0.913, \mathrm{RMSEA}=0.132$, 由于拟合度没有 达到标准, 经过修正得到新的拟合指数为卡方 $=14.125, \mathrm{p}$ $<0.05$, GFI $=0.982$, NFI $=0.963$, CFI $=0.975$, RMSEA $=0.083$ 模型拟合度良好。详见图 1 。

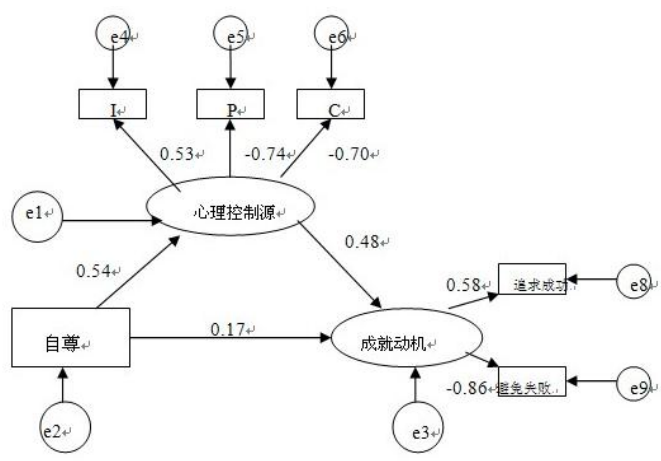

图 1 以心理控制源为中介的路径分析模型

\section{4 讨论}

4.1 高中生自尊、心理控制源和成就动机的性别上的差异分 析

研究发现, 高一学生的自尊、心理控制源和成就动机 在性别变量上均无显著差异, 当前国内外研究显示, 自尊 在性别上基本无显著差异, 与本研究的结果相一致。但在 成就动机这一变量上, 当前国内外很多研究显示男性的成 就动机高于女性, 景怀斌研究发现, 中国女性的成就动机 明显低于男性, 且女性的成就动机较男性更容易受环境激 励因素的影响, 而在原因解释上也合情合理, 由于成就动 机受社会、文化、学校教育、家庭教育等许多因素的影响, 在传统社会规范中, 男孩子与女孩子相比会受到父母、学 校更高的期望和要求，并且男性在步入社会后事业上有更 高的要求, 同时还要成为家庭经济乃至整个家庭的支柱, 责任重大, 因此要求男性追求事业的成功。但本研究未得 出此结果, 原因可能有一下几点: 1、现代社会男女性别逐 渐趋向双性化, 男女受教育程度和机会均等, 思想观念也 大体一致, 女性比过去表现出更多的对社会活动的渴望, 有强烈的参与社会活动的动机。2、由于被试是取自济南市 的一所重点中学, 大部分学生都是来自城市, 而且学习成 绩较同龄人相比优秀, 生活环境、学习环境还有参加活动 都极其相似，所以世界观、人生观、价值观没有太大不同， 
因此在这个比较特别的群体中, 成就动机在男女性别上无 法达到显著性差异也是可以作出解释的。

4.2 高中生自尊、心理控制源和成就动机的相关分析

本研究的相关分析显示, 高一学生的自尊、心理控制 源和成就动机三者之间存在不同程度的统计学上的相关。 高一学生的自尊和成就动机呈现显著的正相关, 自我价值 判断越高, 自我评价越高, 其成就动机越高;倾向于内在归 因的高中生在成就动机上的得分显著高于倾向于归因于他 人掌控和机遇的高中生, 也就是个体越倾向于认为事件的 结果是由内部因素所决定, 越相信自己的行为和能力, 认 为自己能对事情的结果负责, 因此能够主动行动, 不回避 失败, 努力争取, 成就动机也就越高。同样, 采取内部控 制的高中生相信凡事操之于己, 把成功归因于自己的能力 和努力, 把失败归因于自己的努力不足或疏忽。因此在自 尊、心理控制源和成就动机三者的相关研究结果与前文中 提到的李睿等人对大学生的研究相一致。

4.3 高中生自尊、心理控制源和成就动机的路径分析

本研究的回归分析显示, 高中生的自尊对心理控制源 和成就动机具有一定的预测作用, 心理控制源同样也能够 预测成就动机, 因此自尊对成就动机的影响是以心理控制 源为中介在起作用的。如果一个学生的自尊很高, 并且其 心理控制源倾向于内控, 那么我们可以预测其具有高的成 就动机。如果高自尊的学生的心理控制源倾向于他人掌控 或机遇, 那么其成就动机就会比较低。心理控制源是对事 件的归因, 内控性的学生倾向于把事件归因于自身的努力, 因此这样的学生会加强对自己的管理, 更加努力, 其成就 动机也必然高。外控型的学生倾向于事件归因于他人或者 机遇, 因此这样的学生不会付出更多的努力, 其成就动机 也比较低。

\section{5 本研究的不足及展望}

我们所选取的样本仅限于某一中学的高一学生, 样本 代表性不足, 样本容量也较小, 可能会影响结论的推广性,
也在一定程度上影响了数据的结果, 造成了某些结论与以 往研究的差异, 这对本研究的研究深度和广度产生了一定 影响, 但也为后续研究提供依据。

在研究工具上, 本研究使用的是单一的自陈量表, 这 容易受共同方法偏差影响。同时, 被试回答也会出现偏差, 不一定认真的回答问卷。在研究方法上, 本研究使用同一 时间选取信息的横断面研究, 这样无法考察所研究变量间 的动态影响。今后要做进一步的纵向研究, 更加科学的考 察变量间的关系。在今后的研究中, 应该发展出多钟研究 方法, 比如设置实验情境, 对被试的成就动机做深入的考 察。在研究变量上, 本研究涉及的成就动机的相关因素比 较少, 今后需要对成就动机的其他影响因素进行考察, 进 而对高中生的成就动机形成更全面深入的认识。

\section{参考文献(References)}

[1] Atkinson J, Birch D.. An introduction to motivation. New York; Van Nostrand, 1964.

[2] Jerry M.Burger. personality psychology.2004.

[3] Li Rui, Ma Laixiang, Zhang Jinfu. A correlational studu on achievement motivation, locus of control and self-esteem among university students. Health Medicine Research and Practice, Vol.9, No.1, 2002.

[4] Murry H A.. Explorations in personality. New York, Oxford University Press, 1938.

[5] Pang Yu, Tong Yuehua, Tian Lumei. Relationship Between Self-steem, Learning Motivation Academic Achievement. Journal of university of jinan(sci and tech). Vol.25, No.3, 2011.

[6] WANG Y, OLLENDICK T. Across cultural and developmental analysis of self-esteem in Chinese and Western children . Clinical Child and Family Psychology Review, 2001, 4 (3) ,253 -271 .

[7] Zhang Jijia, Chen Jun. A Study on University Students's Achievement Motivation and Fear of Success. Chinese Journal of App;ied Psychology. Vol.8, No.2, 18-22, 2002. 